Pacific Journal of Mathematics

A GENERAL BRAUER-FOWLER THEOREM AND
CENTRALIZERS IN LOCALLY FINITE GROUPS 


\title{
A GENERAL BRAUER-FOWLER THEOREM AND CENTRALIZERS IN LOCALLY FINITE GROUPS
}

\section{Brian HARTLEy}

\begin{abstract}
A classical theorem of Brauer and Fowler, proved by quite elementary arguments, states that the order of a finite non-abelian simple group $G$ is bounded in terms of the order of the centralizer of any involution in $G$. Here we use the classification of finite simple groups to show that the order of such a $G$ is bounded in terms of the order of any automorphism $\alpha$ and the number of fixed points of $\alpha$. It follows easily that if a locally finite group contains an element with finite centralizer, then it has a locally solvable subgroup of finite index.
\end{abstract}

1. Introduction. The main result of this paper is the following.

THEOREM A. There exists an integer-valued function $f(n, k)$ such that if $G$ is a finite group containing an element $x$ of order $n$ such that $\left|C_{G}(x)\right| \leq k$, then $G$ has a soluble normal subgroup of index dividing $f(n, k)$.

It will be useful to say that a quantity is $a, b, \ldots$-bounded, if it is bounded above by some function of $a, b, \ldots$. We shall see that Theorem A follows rather easily from the next result, the proof of which occupies the bulk of the paper.

THEOREM $\mathrm{A}^{\prime}$. Let $G$ be a finite non-abelian simple group admitting an automorphism of order $n$ with at most $k$ fixed points. Then $|G|$ is $n$, $k$-bounded.

This is the general Brauer-Fowler Theorem referred to in the title. In sharp contrast to the real Brauer-Fowler Theorem [5], its proof depends on the classification of finite simple groups. The case of inner automorphisms was dealt with in [14, Theorem A1]. It is unclear whether there is more to be said about the structure of the soluble normal subgroup in Theorem A. Conceivably it should have a subgroup of $n, k$-bounded index and $n$-bounded Fitting height. This is the case when $n$ is a prime power (see Theorem B below). See also [13] for further discussion of this. 
A standard inverse limit argument, given in $\S 2$, establishes the following consequence of Theorem A.

COROLlARY A1. If $G$ is a locally finite group containing an element with finite centralizer, then $G$ has a locally soluble normal subgroup of finite index.

For elements of prime power order, one can say rather more than Theorem A. Taking into account [12], the following may either be deduced from Theorem A, or deduced from Theorem $\mathrm{A}^{\prime}$ by an argument analogous to Brauer-Fong [4]. We leave the details to the reader.

THEOREM B. If $G$ is a finite group containing an element $x$ of prime power order $p^{n}$ with $\left|C_{G}(x)\right| \leq k$, then $\left|G: F_{n} O_{p^{\prime} p}(G)\right|$ is $p^{n}$, $k$ bounded, where $F_{n}(G)$ is the $n$-th term of the Fitting series of $G$.

CoRollaRY B1. If $p$ is a prime and $G$ is a locally finite group containing an element of prime power order $p^{n}$ with finite centralizer, then $G$ contains a normal subgroup $H$ of finite index such that $H=$ $O_{p^{\prime} p}(H)=F_{n}(H)$.

Here, $F_{n}(H)$ is defined inductively by putting $F_{0}(H)=1$ and taking $F_{i+1}(H) / F_{i}(H)$ to be the Hirsch-Plotkin radical of $H / F_{i}(H)$. Corollary B1 is deduced in a similar way to Corollary A1.

The methods used to prove Theorem $\mathrm{A}^{\prime}$ enable us to improve [11] Theorem 2 by removing some exceptions which we were not able to deal with before.

THEOREM C. Let $G$ be an infinite simple group of Lie type over a locally finite field $K$ of characteristic $p$, and let $\alpha$ be an automorphism of finite order $n$ of $G$. Suppose that $p$ does not divide $n$. Then there exist infinitely many primes $q$ such that $\alpha$ fixes an element of order $q$ of $G$.

This was proved in [11] provided that if $G$ has type ${ }^{2} B_{2},{ }^{2} F_{4}$, or ${ }^{2} G_{2}$, or if $n$ is even and $G$ has type $G_{2}$, then $n$ is a prime power. However, we will give a new and simpler proof of the general case here.

An important ingredient in the proof of Theorem $\mathrm{A}^{\prime}$ is the knowledge of centralizers in $G$ of elements in Aut $G \backslash \operatorname{Inndiag} G$, where $G$ is a group of Lie type and Inndiag $G$ the subgroup of Aut $G$ generated by the inner and diagonal automorphisms. Most of these centralizers 
are given in [9], except for involutory "graph automorphisms" in their sense, and most of these have been worked out elsewhere. The case of the even-dimensional orthogonal groups in odd characteristic does not seem to be in the literature in a fully convenient form, though much of it is in Dieudonné [8]. We give a brief discussion of this in $\S 3$.

The work for this paper was carried out at the University of Oregon. The author is grateful to the SERC of the United Kingdom for financial assistance. He also warmly thanks Gary Seitz for much hospitality and kindness, as well as numerous conversations which were essential to the development of the proof.

\section{Deductions from $\mathbf{T h e o r e m} \mathbf{A}^{\prime}$.}

Deduction of Theorem A. It is more convenient (but equivalent) to think of $x$ as an automorphism of $G$ and work in the semidirect product $H=G\langle x\rangle$.

Suppose first that $G=G_{1} \times \cdots \times G_{s}$ is a direct product of nonabelian simple groups $G_{i}$. Then $x$ permutes the direct factors in orbits of lengths dividing $n$. If $G_{i_{1}}, \ldots, G_{i_{t}}$ is such an orbit and $K=G_{i_{1}} \times \cdots \times G_{i_{t}}$, then $C_{K}(x) \cong C_{G_{i_{1}}}\left(x^{t}\right)$. It is a well known consequence of the classification of finite non-abelian simple groups that no such group can admit a fixed-point-free automorphism (see [11] Lemma 4.1 for example). Consequently, $C_{K}(x) \neq 1$. Therefore the number of orbits is at most $k$. Since $\left|C_{G_{i_{1}}}\left(x^{t}\right)\right| \leq k$ we have a bound on $\left|G_{i_{1}}\right|$ from Theorem $\mathrm{A}^{\prime}$, and hence on $|K|$. Consequently, $|G|$ is $n, k$-bounded.

In general, we form part of a chief series of $H$ passing through $G$ and consider a non-abelian chief factor $U / V$. We have $\left|C_{U / V}(x)\right| \leq k$ [2, VIII.10.14], so by what has just been said, $|U / V|$ is $n, k$-bounded. Let $N=\cap C_{G}(U / V)$ over all non-abelian chief factors $U / V$ in our fixed chief series. Then $N$ is soluble. As $G / C_{G}(U / V)$ is embedded in Aut $U / V$, its order is $n, k$-bounded.

Passing to $G / N$, we may assume that $N=1$, and so $G$ has a family $\Lambda$ of $x$-invariant normal subgroups $L$ such that $\bigcap_{L \in \Lambda} L=1$ and $|G / L|$ is $n, k$-bounded for each $L \in \Lambda$. Now we use induction on $k$. If $k=1$, then $G$ is soluble as we have remarked. Otherwise we can choose $L \in \Lambda$ such that $C_{G}(x) \not L$. Then by induction, $L$ has a soluble normal subgroup $S$ of $n, k / 2$-bounded index, and since $|G / L|$ is $n, k$-bounded, so is $|G: S|$.

Deduction of Corollary A1. Let $G$ be a locally finite group containing an element $x$, of order $n$ say, with finite centralizer of order $k$. 
By Theorem $\mathrm{A}$, we have a number $f$ such that if $F$ is any finite subgroup of $G$ containing $x$, then $F$ contains a soluble normal subgroup of index at most $f$. Let $\Sigma(F)$ be the set of all such subgroups of $F$ and $\Phi$ the set of all finite subgroups of $G$ containing $x$. If $F_{i} \in \Phi(i=1,2), F_{1} \geq F_{2}$, and $S \in \Sigma\left(F_{1}\right)$, then $S \cap F_{2} \in \Sigma\left(F_{2}\right)$. Thus $\pi_{F_{1} F_{2}}: S \mapsto S \cap F_{2}$ maps $\Sigma\left(F_{1}\right)$ into $\Sigma\left(F_{2}\right)$. We have an inverse system $\Sigma(F)_{F \in \Phi}$ of finite non-empty sets. The inverse limit is nonempty [15], and so we can choose $S_{F} \in \Sigma(F)$, one for each $F \in \Phi$, such that $S_{F_{1}} \cap F_{2}=S_{F_{2}}$ whenever $F_{1} \geq F_{2}$. Let $S=\bigcup_{F \in \Phi} S_{F}$. It is easy to see that this is a locally soluble normal subgroup of $G$ of index at most $f$

3. Some background results. Because of the classification of finite simple groups, which tells us among other things that there are only finitely many sporadic groups, the latter can be ignored in proving Theorem $\mathrm{A}^{\prime}$. This leaves us with alternating groups and groups of Lie type. The alternating groups are easy to handle and will be left to the reader. We now set up terminology for groups of Lie type and assemble some basic facts, many of which will be well known. For general background in this area, see $[3,7,17]$.

Notation. $\bar{G}$ denotes a connected reductive algebraic group over an algebraically closed field $K$ of characteristic $p>0$. Let $\sigma$ be a Frobenius map on $\bar{G}$ in the sense of [7, p. 31]. When $\bar{G}$ is semisimple, this means precisely that $\sigma$ is a surjective algebraic endomorphism of $\bar{G}$ with finite fixed point group $\bar{G}_{\sigma}$. Sometimes we denote the fixed point group by $C_{\bar{G}}(\sigma)$. Let $G=O^{p^{\prime}}\left(\bar{G}_{\sigma}\right)$. It is well known that $\sigma$ fixes a Borel subgroup $B$ of $\bar{G}$ and a maximal torus $T$ of $\bar{G}$ contained in $B$. Let $N=N_{\bar{G}}(T)$ and $W=N / T$ be the Weyl group. Let $\sigma^{*}$ be the map induced by $\sigma$ on the character group $X=X(T)$ and on its real extension $X_{R}=X \otimes R$. There is a unique smallest integer $\delta \geq 1$ such that $\sigma^{* \delta}$ is a positive integral multiple of the identity, and we can write then $\sigma^{*}=q \sigma_{0}$, where $q=q(\sigma)$ is a positive real number and $\sigma_{0}^{\delta}=1$. See [7], also [14, Section 3].

Suppose now that $\bar{G}$ is simple as algebraic group. . In this case, $\sigma_{0}$ is an isometry of $X_{R}[17, \mathrm{p}$. E21]. The number $q$ is an integral power of $p$ except in types ${ }^{2} B_{2},{ }^{2} F_{4},{ }^{2} G_{2}$, when $q^{2}$ is an odd power of 2,2 or 3 respectively, and $G$ is considered as defined over $F_{q}$ except in the three cases just mentioned, when it is considered as defined over $F_{q^{2}}$ (see [7, p. 36]). We often think of $G$ as a Chevalley group constructed from a complex simple Lie algebra. Let Chev denote the 
collection of all such groups $G$. If $\bar{G}$ is also of adjoint type, then $G$ is simple except in a few small cases, and all finite simple groups of Lie type arise in this way.

Our notation and terminology for automorphisms of $G$ is sometimes consistent with [6], [19] and sometimes with [9, p. 78 et seq]. This is unfortunate since their terminologies are sometimes in conflict, especially as regards graph automorphisms, but seems inescapable. When we use the undecorated terms "graph automorphism" and "field automorphism", we do so in the sense of [6], [19]. In particular, we have in mind a fixed parametrization of $G$ as a Chevalley group. We write "GL-graph automorphism" (for Gorenstein-Lyons), when we wish to speak of a graph automorphism in the sense of [9]. We have (see [9])

$$
A=\text { Aut } G=\bar{G}_{\sigma} \Phi_{G} \Gamma_{G},
$$

where we have identified $\bar{G}_{\sigma}$ with the group Inndiag $G$ it induces on $G$ by conjugation, $\Phi_{G}$ is the group of field automorphisms of $G$, and $\Gamma_{G}$ is a canonical set of graph automorphisms constructed as in $[19, \mathrm{p}$. 157] or [6], together with, and perhaps consisting only of, the identity map. There is uniqueness of expression in the above.

We do not need to explain fully the terminology of [9], except to clarify the term "GL-graph automorphism". An element $\alpha$ is a GLgraph automorphism if and only if either (i) $G$ is untwisted and not of type $B_{2}, F_{4}$, or $G_{2}$, and $\alpha \in \bar{G}_{\sigma} \Gamma_{G} \backslash \bar{G}_{\sigma}$, or (ii) $G$ is twisted, the associated twist, or symmetry of the Dynkin diagram, has order $d$, and $\alpha$ is an element of $A \backslash \bar{G}_{\sigma}$ of order divisible by $d$. This is not quite what is said, but appears to be what is intended, in [9]. In particular, the groups $B_{2}, F_{4}$ and $G_{2}$ have no GL-graph automorphisms. We need the following, which in most cases is well known.

LEMMA 3.1. Suppose that $\bar{G}$ is simple of adjoint type. Let $\alpha$ be an element of prime order $r$ in $A \backslash \bar{G}_{\sigma}$. Then $C_{G}(\alpha)$ involves a finite non-abelian simple group $H$ such that $|G|$ is $|H|$, r-bounded.

Proof. This follows from [9, (9.1)] except in the case when $r=2$ and $\alpha$ is a GL-graph automorphism. When $G$ is untwisted this means that $\alpha \in \bar{G}_{\sigma} \gamma \backslash \bar{G}_{\sigma}$, where $\gamma$ is an element of order 2 in $\Gamma_{G}$, and $G$ has type $A_{l}, D_{l}$ or $E_{6}$. In characteristic 2 these cases are covered in [1]. Recalling that the graph automorphism of $P \Omega^{+}(2 l, q)$ is induced by conjugation with an involution in $O^{+}(2 l, q)[6$, p. 272], we find the case $D_{l}$ in $[1,(8.7),(8.10)]$. Types $A_{l}$ and $E_{6}$ are in $[1,(19.8)$, 
see also $\S 13]$. For types $A_{l}$ and $E_{6}$ in odd characteristic, see [10, Lemmas 4.25 and 4.27 and Remark 4.28]. The orthogonal groups $D_{l}$ in odd characteristic do not appear to be covered in the literature and we deal with them in Lemma 3.2 below.

When $G$ is twisted, the statement that $\alpha$ is a GL-graph automorphism of order 2 means that $\alpha \in \bar{G}_{\sigma} \gamma \backslash \bar{G}_{\sigma}$, where $\gamma$ is the element of order 2 in $\Phi_{G}$, and $G$ has type ${ }^{2} A_{l},{ }^{2} D_{l},{ }^{2} E_{6}$. In characteristic 2 we consult $[1,(8.7),(19.8)]$. In odd characteristic, types ${ }^{2} A_{l}$ and ${ }^{2} E_{6}$ are dealt with in [10], see Lemmas 4.26 and 4.29 and Remark 4.30. This leaves the orthogonal groups ${ }^{2} D_{l}$ in odd characteristic, and they are also dealt with in Lemma 3.2 below.

We now set up some background on the orthogonal groups, in preparation for Lemma 3.2.

For some of what follows, and general background, see [8]. Let $S=\operatorname{SO}(2 l, K)$, where $K$ is an algebraically closed field of odd characteristic $p>0$. Then $S$ is a group of invertible linear transformations of a $2 l$-dimensional vector space $V$ over $K$, preserving a nondegenerate symmetric bilinear form $f$ on $V$. Since all such forms are equivalent, we may take $f$ to have matrix

$$
E_{2 l}=\left(\begin{array}{cc}
0 & I_{l} \\
I_{l} & 0
\end{array}\right)
$$

with respect to a suitable basis $x_{1}, \ldots, x_{l}, x_{-1}, \ldots, x_{-l}$ of $V$. Let $\gamma$ be given by

$$
\gamma\left(x_{ \pm l}\right)=x_{\mp l} ; \quad \gamma\left(x_{j}\right)=x_{j} \quad(j \neq l) .
$$

Then $\gamma$ preserves $f$, and so $\gamma \in O(2 l, K)$. From [6, p. 272], we have that conjugation with $\gamma$ induces on $S O(2 l, K)$ its canonical graph automorphism (there is a natural root structure in this situation). We have

$$
O=O(2 l, K)=S O(2 l, K) \rtimes\langle\gamma\rangle .
$$

Let $q$ be a power of $p$, think of $G$ as a matrix group with respect to the above choice of basis, and let $\sigma$ be the $q$-th power map on $S$. Then $\sigma$ and $\sigma \gamma$ are Frobenius maps on $S$ and induce Frobenius maps on $\bar{G}=\operatorname{PSO}(2 l, K)$. The group $\bar{G}$ is the adjoint group of type $D_{l}$, and

$$
O^{p^{\prime}}\left(\bar{G}_{\tau}\right) \cong P \Omega^{ \pm}(2 l, q),
$$

where the plus sign is taken if $\tau=\sigma$ and the minus sign if $\tau=$ $\sigma \gamma$. We are interested in the centralizers in $O^{p^{\prime}}\left(\bar{G}_{\tau}\right)$ of involutions in $\bar{G}_{\tau}\langle\gamma\rangle$, and in particular in the non-abelian composition factors of these centralizers. 
LeMma 3.2. Let $\bar{G}$ be as above, with $l>1$, let $w$ be any involution in $\bar{G}_{\tau}\langle\gamma\rangle$, and let $G=O^{p^{\prime}}\left(\bar{G}_{\tau}\right)$. Then $C_{G}(w)$ involves at least one (and at most two) of the groups

$$
\begin{aligned}
& P \Omega^{ \pm}\left(l, q^{2}\right), \operatorname{PSL}(l, q), \operatorname{PSU}(l, q), P \Omega^{ \pm}(2 l-1, q), \\
& P \Omega^{\varepsilon_{1}}(d, q) \times P \Omega^{\varepsilon_{2}}(2 l-d, q) \quad\left(\varepsilon_{i} \in\{ \pm 1\}, 1<d<l\right) .
\end{aligned}
$$

More detail can be extracted from the proof, but we have no need of it.

Proof. Since $\bar{G}_{\tau} / G$ is of order dividing 4 [6, p. 99], it suffices to show that $C_{\bar{G}}(w)$ involves one of the above groups. Let $Z$ be the centre of $S$. Then $Z=\langle-1\rangle$. For any element $x \in O$, let

$$
C^{*}(x)=\left\{g \in S: x^{g}= \pm x\right\} .
$$

Then $C^{*}(x) / Z=C_{\bar{G}}(x Z)$. We calculate $C^{*}(w)$ for each element $w \in O$ such that $w Z$ has order 2. If $w Z$ is $\tau$-invariant and $C=$ $C^{*}(w) / Z$, then

$$
C_{\bar{G}_{\tau}}(w Z)=C_{\tau}
$$

and our results will follow from standard facts about Frobenius maps.

Now $w Z$ is an involution if and only if $w$ is either an involution different from -1 , or an element of order 4 whose square is -1 .

Suppose first that $u$ is an involution, $u \neq-1$. Then we have

$$
V=V_{-} \oplus V_{+},
$$

where $V_{-}$and $V_{+}$are respectively the -1 and +1 -eigenspaces of $u$. These eigenspaces are orthogonal, and so the restriction of $f$ to them is non-degenerate. Let $d=\operatorname{dim} V_{-}$, and $C^{*}=C^{*}(u)$. Then $C^{*}$ leaves $V_{-}$and $V_{+}$invariant if $d \neq l$, and permutes them if $d=l$. We clearly have

$$
C^{*}(u)=(O(d, K) \times O(2 l-d, K)) \cap S \quad(d \neq l),
$$

and

$$
C^{*}(u)=\left(O(l, K) \curlywedge C_{2}\right) \cap S \quad(d=l) .
$$

Some abuse of notation is involved on the right hand side, but it should be clear what is intended.

Now let $v$ be an element of order 4 in $O$ such that $v^{2}=-1$. Let $\zeta$ be a primitive 4-th root of 1 in $K$. Then

$$
V=V_{\zeta} \oplus V_{-\zeta}
$$


is the direct sum of the $\zeta$ - and - $\zeta$-eigenspaces of $v$. These are easily seen to be totally isotropic, and hence by Witt's Theorem they have dimension at most $l$. Therefore $\operatorname{dim} V_{\zeta}=\operatorname{dim} V_{-\zeta}=l$. Each of these spaces induces the full dual of the other with respect to $f$. An element of $C^{*}(v)$ either fixes both $V_{\zeta}$ and $V_{-\zeta}$ or interchanges them, and in the former case, its actions on these two spaces must be mutually dual. We see that

$$
C^{*}(v)=(\mathrm{GL}(l, K)\langle t\rangle) \cap S,
$$

where $t$ is an element of order 2 interchanging $V_{\zeta}$ and $V_{-\zeta}$ and inducing the inverse transpose map on $\mathrm{GL}(l, K)$.

It follows that the semisimple part of $C^{*}(u) / Z$ is either a central product (possibly direct) of two special orthogonal groups whose degrees sum to $2 l$, or $\operatorname{SO}(2 l-1, K)$ in case $d=1$ or $2 l-1$, and that of $C^{*}(v) / Z$ is a central extension of $\operatorname{PSL}(l, K)$.

Now if $w$ is any involution in $\bar{G}_{\tau}\langle\gamma\rangle$, then $w$ has the form $u$ or $v$ above, and $w Z$ is $\tau$-invariant. Hence $C=C^{*}(w) / Z$ is $\tau$-invariant, and $\tau$ induces a Frobenius map on it.

By standard facts, the perfect radical of $C_{\tau}$ will be a central product of one or two central extensions of finite versions (possibly twisted) of the simple components of $C^{*} / Z$, defined over the field of $q$ or $q^{2}$ elements. See [14], Lemma 3.3 and proof of Theorem C: finite case. The result follows.

The next result can be found for maximal tori of semisimple groups in $[16,(2.4)]$, but the present case needs a little work and seems worth spelling out. Note that the possiblity $S=\bar{G}$ is allowed.

LEMMA 3.3. Let $\bar{G}$ be connected reductive, and $S$ be an arbitrary non-trivial $\sigma$-invariant torus of $\bar{G}$. Let $S$ have rank $r$. Then $\left|S_{\sigma}\right|=$ $|f(q)|$, where $f(X)$ is a real monic polynomial of degree $r$ whose roots are roots of 1 of degree at most $r^{2}$ over the rationals. If $\bar{G}$ is simple, then $r^{2}$ can be replaced by $2 r$. Hence

$$
(q-1)^{r} \leq\left|S_{\sigma}\right| \leq(q+1)^{r} .
$$

Proof. The map $\sigma$ induces a Frobenius map on $C_{\bar{G}}(S) / S$ and fixes a maximal torus $U / S$ of this group [8, p. 33]. Then $U$ is a $\sigma$-invariant maximal torus of $\bar{G}$. Let $W$ be the character group of $U$, and

$$
S^{\perp}=\{\chi \in W: \chi(s)=1 \text { for all } s \in S\} .
$$

Then $\bar{W}=W / S^{\perp}$ is naturally isomorphic to the character group $X(S)$ of $S[8, \mathrm{p} .26]$. Let $\sigma^{*}$ be the map induced on the character group 
of $W$ by $\sigma$, so that

$$
\sigma^{*} \chi(u)=\chi(\sigma(u)) \text { for } \chi \in W, u \in U .
$$

Then $S^{\perp}$ is $\sigma^{*}$-invariant, and $S_{\sigma}$ is in duality with, and hence isomorphic to, $\bar{W} /\left(\sigma^{*}-1\right) \bar{W}$. By standard facts, the order of this group is $\left|\operatorname{det}\left(\sigma^{*}-1\right)_{\bar{W}}\right|$, where $\left(\sigma^{*}-1\right)_{\bar{W}}$ is the endomorphism induced on $\bar{W}$ by $\sigma^{*}-1$. In calculating this determinant, we can replace $\bar{W}$ by its real extension $\bar{W}_{R}$. Then $\sigma^{*}$ takes the form $q \sigma_{0}$, where $\sigma_{0}$ has finite order. For this is true of a maximally split torus, as is well known, and in general we twist by an element of the Weyl group, which just modifies $\sigma_{0}$ by an isometry of finite order. Thus, if $\bar{\sigma}_{0}$ is the map induced on $\bar{W}_{R}$ by $\sigma_{0}$, then

$$
\begin{aligned}
\left|S_{\sigma}\right| & =\left|\operatorname{det}\left(q \bar{\sigma}_{0}-1\right)\right|=\left|\operatorname{det}\left(q-\bar{\sigma}_{0}^{-1}\right)\right| \cdot\left|\operatorname{det} \bar{\sigma}_{0}\right| \\
& =\left|\operatorname{det}\left(\bar{\sigma}_{0}^{-1}-q\right)\right|=|f(q)|,
\end{aligned}
$$

where $f$ is the characteristic polynomial of $\bar{\sigma}_{0}^{-1}$. Since $\bar{\sigma}_{0}^{-1}$ has finite order, the roots of $f$ are roots of 1 . We have $f(X)=\prod_{i=1}^{r}\left(X-\zeta_{i}\right)$, where the $\zeta_{i}$ are roots of 1 , hence $(q-1)^{r} \leq|f(q)| \leq(q+1)^{r}$, by the triangle inequality.

It remains only to give a (probably rather crude) estimate for the degrees over $Q$ of the eigenvalues of $\sigma_{0}$. If $\zeta$ is such an eigenvalue, then $q \zeta$ is an eigenvalue of $\sigma^{*}$, so $q \zeta$ is an algebraic integer of degree at most $r$ over $Q$. Taking the absolute value of its norm, we find that $q^{t} \in Z$ for some $t \leq r$, whence $\zeta^{t}$ has degree at most $r$ over $Q$. Hence $\zeta$ has degree at most $r^{2}$ over $Q$. If $\bar{G}$ is simple, then $q^{2}$ is an integer, and the above argument yields $2 r$ instead of $r^{2}$.

Much, if not all, of the next result is well known.

LEMMA 3.4. Let $\bar{G}$ be simple of adjoint type and let $s$ be a semisimple element of order $n$ in $\bar{G}_{\sigma}$. Then $C_{G}(s)$ contains a product $Y_{1} \cdots Y_{t}$ of pairwise commuting groups in $\mathbf{C h e v}$, defined over extension fields of that over which $G$ is defined. If $\bar{G}$ is of classical type and has rank $l$, and

$$
r(n)=n+\left[\frac{4}{n}\right] \text {, }
$$

then for some $i$, the overlying algebraic group of $Y_{i}$ has rank at least $(l-n) / r(n)$. Further, $t \geq 1$ unless $C_{\bar{G}}(s)^{0}$ is abelian, in which case $C_{\bar{G}}(s)$ contains no element of order $p$.

Proof. Let $\bar{G}_{s c}$ be the universal cover of $\bar{G}$ and $\pi: \bar{G}_{s c} \longrightarrow \bar{G}$ the canonical projection. Then $s$ is the image of a semisimple element 
$s^{\prime} \in \bar{G}_{s c}$, and $\pi$ maps $C_{\bar{G}_{s c}}\left(s^{\prime}\right)$ onto $C_{\bar{G}}(s)^{o}$. The structure of $C_{\bar{G}_{s c}}\left(s^{\prime}\right)$ is given by [14, Theorem 4.2], and then applying [14, Lemma 3.3] to $\left(C_{\bar{G}}(s)^{o}\right)_{\sigma}$ gives the result.

See also [16, (2.9)].

4. Generalities on fixed points of automorphisms of finite groups of Lie type. We continue with the notation of $\S 3$, and assume throughout that $\bar{G}$ is simple (but not necessarily of adjoint type). We think of $\bar{G}$ as realized in some explicit way as a Chevalley group over $K$. Let $\Phi$ be the group of field automorphisms of $\bar{G}$ and $\Gamma$ the set of graph automorphisms constructed as in $[6, \S 12.2-12.4]$, together with the identity.

The first two results are very similar, but it seems better to deal with the cases of $B_{2}, F_{4}$ and $G_{2}$ separately.

Proposition 4.1. Suppose that $\bar{G}$ is not of type $B_{2}, F_{4}$ or $G_{2}$. Let $\sigma=\nu \gamma$, where $\nu$ is a Frobenius map in $\Phi$ and $\gamma \in \Gamma$, and let $G=O^{p^{\prime}}\left(\bar{G}_{\sigma}\right)$. Let $\alpha=g \phi \delta \in A=$ Aut $G$, with $g \in \bar{G}_{\sigma}, \phi \in \Phi_{G}$, and $\delta \in \Gamma_{G}$. Let $\phi$ have order $r$, let $\alpha$ have order $n$, and let $q=q(\sigma)$. Then one of the following occurs.

(I) $C_{\bar{G}_{\sigma}}(\alpha)=C_{\bar{G}}\left(g_{1}\right)_{\mu}$, where $g_{1}=\alpha^{r} \in \bar{G}_{\sigma} \Gamma_{G}$, and $\mu$ is a Frobenius map on $\bar{G}$ such that $\mu\left(g_{1}\right)=g_{1}$ and $q(\mu)^{r}=q$. Further, $g_{1}$ has order $n / r$.

(II) $\gamma \neq 1, \delta=1$, and the order $m$ of $\gamma$ divides $r$. Let $r=m s$, let $\alpha^{s}=g_{2} \phi^{s}$, where $g_{2} \in \bar{G}_{\sigma}$, and let $\gamma^{\prime}$ be the element of $\Gamma$ which agrees with $\phi^{s}$ on $G$. Then there is a Frobenius map $\mu$ on $\bar{G}$ such that $\mu\left(g_{2} \gamma^{\prime}\right)=g_{2} \gamma^{\prime}, q(\mu)^{s}=q$, and $C_{\bar{G}_{\sigma}}(\alpha)=C_{G}\left(g_{2} \gamma^{\prime}\right)_{\mu}$. Here, the order of $g_{2} \gamma^{\prime}$ is $n / s$.

Further, if $r=n$ and $n$ is coprime to the order of $\gamma$ (the latter happens in particular if $\gamma=1$ ), then $\alpha$ is conjugate under $\bar{G}_{\sigma}$ to an element of $\Phi_{G} \Gamma_{G}$.

In case (II), of course, $\gamma^{\prime}=\gamma$ unless $\bar{G}$ has type $D_{4}$, in which case $\gamma^{\prime}=\gamma^{ \pm 1}$.

The analogous result for types $B_{2}, F_{4}$ and $G_{2}$ is as follows.

Proposition 4.2. Suppose that $\bar{G}$ is of type $B_{2}, F_{4}$ or $G_{2}$. Let $\sigma=\nu \gamma$, where $\nu$ is a Frobenius map in $\Phi$ and $\gamma \in \Gamma$, and let $G=O^{p^{\prime}}\left(\bar{G}_{\sigma}\right)$. Let $\alpha=g \phi \in A=$ Aut $G$, with $g \in \bar{G}_{\sigma}$ and $\phi \in \Phi_{G} \Gamma_{G}$. Let $\phi$ have order $r$ and $\alpha$ have order $n$, and let $g_{1}=\alpha^{r} \in \bar{G}_{\sigma}$. Then

$$
C_{\bar{G}_{\sigma}}(\alpha)=C_{\bar{G}}\left(g_{1}\right)_{\theta} \text {, }
$$


where $\theta$ is a Frobenius map on $\bar{G}$ such that $\theta\left(g_{1}\right)=g_{1}$ and $q(\theta)^{r}=$ $q$. Further, $g_{1}$ has order $n / r$, and if $r=n$, then $\alpha$ is conjugate under $\bar{G}_{\sigma}$ to an element of $\Phi_{G} \Gamma_{G}$.

Special cases of the above results, at least, are certainly known, see [9] for example. Lang's Theorem is the main ingredient in their proof. It is useful to separate out part of the argument as follows.

LemmA 4.3. Let $\alpha=g \theta \in A$, with $g \in \bar{G}_{\sigma}$ and $\theta \in \Phi_{G} \Gamma_{G}$. Let $\theta$ have order $r$ and $\alpha^{r}=(g \theta)^{r}=g_{1} \in \bar{G}_{\sigma}$. Suppose there is a Frobenius map $\tau$ on $\bar{G}$ that induces $\theta$ on $\bar{G}_{\sigma}$ and satisfies $\tau^{r}=\sigma$. Then

$$
C_{\bar{G}_{\sigma}}(\alpha)=C_{\bar{G}}\left(g_{1}\right)_{\psi},
$$

where $\psi$ is a Frobenius map on $\bar{G}$ such that $\psi\left(g_{1}\right)=g_{1}$ and $\psi$ is $\bar{G}$-conjugate to $\tau$.

If, further, $r=n$, then $\alpha$ is conjugate to $\theta$ under $\bar{G}_{\sigma}$.

Proof. We have

$$
C_{\bar{G}_{\sigma}}(\alpha)=C_{\bar{G}}(\langle\sigma, g \tau\rangle)=C_{\bar{G}}\left(\left\langle\tau^{r}, g \tau\right\rangle\right) .
$$

Now

$$
(g \tau)^{r}=g \cdot g^{\tau^{-1}} \cdots g^{\tau^{-(r-1)}} \tau^{r}=g \cdot g^{\theta^{-1}} \cdots g^{\theta^{-(r-1)}} \tau^{r}=g_{1} \tau^{r},
$$

whence $\tau^{r}=g_{1}^{-1}(g \tau)^{r}$. So

$$
C_{\bar{G}_{\sigma}}(\alpha)=C_{\bar{G}}\left(\left\langle g_{1}^{-1}(g \tau)^{r}, g \tau\right\rangle\right)=C_{\bar{G}}\left(\left\langle g_{1}, g \tau\right\rangle\right) .
$$

By Lang's Theorem, working in the split extension $\bar{G} \Phi \Gamma$, we have that $g \tau$ is $\bar{G}$-conjugate to $\tau$. Also, $g \tau$ acts as $\alpha$ on $\bar{G}_{\sigma}$ and so commutes with $\alpha^{r}=g_{1}$. Letting $\psi$ be the Frobenius map induced by conjugation with $g \tau$, we deduce that

$$
C_{\bar{G}_{\sigma}}(\alpha)=C_{\bar{G}}\left(g_{1}\right)_{\psi} \text {. }
$$

Now suppose that $r=n$. Choose $h \in \bar{G}$ such that

$$
(g \tau)^{h}=\tau .
$$

Raising (4.2) to the $r$-th power and using (4.1) shows that $h$ commutes with $\tau^{r}=\sigma$, that is, $h \in \bar{G}_{\sigma}$. Then we find that

$$
\alpha^{h}=(g \theta)^{h}=g^{h}\left[h, \theta^{-1}\right] \theta=g^{h}\left[h, \tau^{-1}\right] \theta=\theta
$$

from (4.2). 
Proof of Proposition 4.1. We consider various cases.

Case 1. $\gamma=1, o(\delta) \mid r$. Let $\tau_{0}$ be the element of $\Phi$ such that $\tau_{0}^{r}=\sigma$. Then $\tau_{0}$ induces an element $\phi_{0}$ of $\Phi_{G}$ of order $r$. Hence $\left\langle\phi_{0}\right\rangle=\langle\phi\rangle$, and $\phi_{0}=\phi^{s}$ with $(r, s)=1$. Write $\alpha=\alpha_{1} \alpha_{2}$ where $\alpha_{1}$ is an $r$-element, $\alpha_{2}$ is an $r^{\prime}$-element, and $\alpha_{1} \alpha_{2}=\alpha_{2} \alpha_{1}$. We have $\left\langle\alpha_{1}^{s}, \alpha_{2}\right\rangle=\langle\alpha\rangle$ and so if $\beta=\alpha_{1}^{s} \alpha_{2}$, then $C_{\bar{G}_{\sigma}}(\beta)=C_{\bar{G}_{\sigma}}(\alpha)$. We have $\beta=g^{*} \phi^{s} \delta^{s}$ with $g^{*} \in \bar{G}_{\sigma}$. Let $\tau=\tau_{0} \delta^{s}$. Since $\delta^{r}=1$, we have $\tau^{r}=\sigma$, and $\tau$ induces $\phi^{s} \delta^{s}$. Clearly $\left\langle\beta^{r}\right\rangle=\left\langle\alpha^{r}\right\rangle$. By Lemma 4.3,

$$
C_{\bar{G}_{\sigma}}(\beta)=C_{\bar{G}}\left(\beta^{r}\right)_{\mu}=C_{\bar{G}}\left(g_{1}\right)_{\mu},
$$

where $\mu$ is a Frobenius map $\bar{G}$-conjugate to $\tau$ and $g_{1}$ is $\mu$-invariant. Thus, $q(\mu)^{r}=q$. Lemma 4.3 also yields that if $r=n$ in this case, then $\beta$, and hence $\alpha$, is $\bar{G}_{\sigma}$-conjugate to an element of $\Phi_{G} \Gamma_{G}$.

Case 2. $\gamma=1, \delta \neq 1$, and $(o(\delta), r)=1$. Note that in this case, $r \neq n$. Let $\tau \in \Phi$ be such that $\tau^{r}=\sigma$. Then $\tau$ induces an element of order $r$ of $\Phi_{G}$, and by modifying the $r$-part of $\alpha$ as in Case 1 , we may assume that this is $\phi$. Let $m=o(\delta)$. We can write $\langle\alpha\rangle=\left\langle\alpha_{1}\right\rangle \times\left\langle\alpha_{2}\right\rangle$, where $\alpha_{1}$ is an $m^{\prime}$-element, $\alpha_{2}$ is an $m$-element, and $\alpha_{1}=x \phi, \alpha_{2}=y \delta$ with $x, y \in \bar{G}_{\sigma}$. Then

$$
\left\langle\alpha^{r}\right\rangle=\left\langle a_{1}^{r}, \alpha_{2}\right\rangle \text {. }
$$

Let $x_{1}=\alpha_{1}^{r} \in \bar{G}_{\sigma}$. Then $(x \tau)^{r}=x_{1} \tau^{r}$ as in Case 1, so $\tau^{r}=x_{1}^{-1}(x \tau)^{r}$. We have

$$
\begin{aligned}
C_{\overline{G_{\sigma}}}(\alpha) & =C_{\bar{G}}\left(\left\langle\tau^{r}, x \tau, y \delta\right\rangle\right)=C_{\bar{G}}\left(\left\langle x_{1}^{-1}(x \tau)^{r}, x \tau, y \delta\right\rangle\right) \\
& =C_{\bar{G}}\left(\left\langle x_{1}, x \tau, y \delta\right\rangle\right)=C_{\bar{G}}\left(\left\langle\alpha_{1}^{r}, \alpha_{2}, x \tau\right\rangle\right)=C_{\bar{G}}\left(g_{1}\right)_{\mu},
\end{aligned}
$$

from (4.3), where $\mu=x \tau$ which, by Lang's Theorem, is $\bar{G}$-conjugate to $\tau$. Since $x \tau=\mu$ acts on $g_{1}$ as $x \phi=\alpha_{1}$, certainly $g_{1}$ is $\mu$ invariant, and $q(\mu)^{r}=q$.

Case 3. $\gamma \neq 1,(r, m)=1$, where $m=o(\gamma) \in\{2,3\}$. In this case $\delta=1$. We wish to invoke Lemma 4.3. Let $\tau \in \Phi$ be such that $\tau^{r}=\nu$, and let $\nu$ induce $\nu_{1} \in \Phi_{G}$, so that $\nu_{1}$ has order 2 or 3 . First suppose that $\gamma$ has order 2. Then $\nu_{1}$ has order 2. Consider the effect of $\tau \gamma$ on $\bar{G}_{\sigma}$. Now $\tau^{r}$ induces $\nu_{1}$ on $\bar{G}_{\sigma}$, and since $\nu \gamma$ is trivial on $\bar{G}_{\sigma}, \gamma$ induces $\nu_{1}$ also. So $\tau \gamma$ induces a field automorphism of order $r$ on $\bar{G}_{\sigma}$, and by adjusting the $r$-part of $\alpha$, we may assume that $\tau \gamma$ induces $\phi$. Now everything, including the final conjugacy statement if $r=n$, follows from Lemma 4.3.

When $\gamma$ has order 3 the situation is broadly similar. Arguing as above, we find that $(\tau \gamma)^{r}$ induces $\nu_{1}^{1-r}$ on $\bar{G}_{\sigma}$. If $r \equiv 1 \bmod 3$, then 
we find that $\tau \gamma$ induces a field automorphism of order $r$ on $\bar{G}_{\sigma}$, and everything is identical. If $r \equiv-1 \bmod 3$, we consider $\tau \gamma^{2}$ instead.

Case 4. $\gamma \neq 1, m=o(\gamma) \mid r$. Again $\delta=1$. Verification of the final conjugacy statement does not arise here. Set $r=m s$, and let $\tau$ be the element of $\Phi$ such that $\tau^{s}=\nu$. Then $\tau$ induces on $\bar{G}_{\sigma}$ a field automorphism of order $m s=r$, which is the order of $\phi$. Now the natural map $\bar{G}_{\sigma} \Phi \Gamma \rightarrow \bar{G}_{\sigma} \Phi_{G}$ is monomorphic on the normal subgroup $\bar{G}_{\sigma} \Gamma$, and the element $g_{2} \gamma^{\prime}$ occurring in (II) is the unique element of $\bar{G}_{\sigma} \Gamma$ mapping to $\alpha^{s}$ under it. Thus, replacing $\alpha$ by another generator of $\langle\alpha\rangle$ does not change $\left\langle g_{2} \gamma\right\rangle$. This allows us to make such a replacement and assume that $\tau$ induces $\phi$ on $\bar{G}_{\sigma}$. Now we have $\gamma^{\prime}=\gamma^{-1}$, since $\tau^{s}=\nu, \tau^{s}$ induces $\phi^{s}$, and $\nu \gamma$ induces the identity on $\bar{G}_{\sigma}$. We have

$$
(g \tau)^{s}=g \cdot g^{\tau^{-1}} \cdots g^{\tau^{-(s-1)}} \tau^{s}=g \cdot g^{\phi^{-1}} \cdots g^{\phi^{-(s-1)}} \tau^{s}=g_{2} \tau^{s},
$$

since $(g \phi)^{s}=g_{2} \phi^{s}$. Hence $\nu=\tau^{s}=g_{2}^{-1}(g \tau)^{s}$ and

$$
\begin{aligned}
C_{\bar{G}_{\sigma}}(\alpha) & =C_{\bar{G}}\left(\left\langle\gamma g_{2}^{-1}(g \tau)^{s}, g \tau\right\rangle\right) \\
& =C_{\bar{G}}\left(\left\langle g_{2} \gamma^{-1}, g \tau\right\rangle\right)=C_{\bar{G}}\left(\left\langle g_{2} \gamma^{\prime}, g \tau\right\rangle\right) .
\end{aligned}
$$

Now $g_{2} \gamma^{\prime}$ has order $n / s$ and commutes with $g \tau$, since these statements become true after applying the natural map $\bar{G}_{\sigma} \Phi \Gamma \rightarrow \bar{G}_{\sigma} \Phi_{G}$, and by Lang's Theorem, $g \tau$ induces on $\bar{G}$ a Frobenius map $\mu$ conjugate to $\tau$ under $\bar{G}$. Therefore $q(\mu)^{s}=q\left(\tau^{s}\right)=q(\nu)=q(\sigma)$, and $C_{\bar{G}_{\sigma}}(\alpha)=C_{\bar{G}}\left(g_{2} \gamma^{\prime}\right)_{\mu}$.

Proof of Proposition 4.2. This follows directly from Lemma 4.3, since every element of $\Phi_{G} \Gamma_{G}$ is induced in the appropriate way by a Frobenius map on $\bar{G}$, when $\bar{G}$ has type $B_{2}, F_{4}$ or $G_{2}$.

Now we extract from the above results the information that we need for the proofs of Theorems $A^{\prime}$ and $C$.

THEOREM 4.4. Let $\bar{G}$ have rank $l$, let $\alpha$ be an automorphism of order $n$ of $G$, and suppose that $p$ does not divide $n$. Then

(i) $C_{\bar{G}_{\sigma}}(\alpha)=H_{\psi}$, where $H$ is an infinite reductive group and $\psi$ is a Frobenius map on $\bar{G}$ such that $H$ is $\psi$-invariant and $q(\psi)^{m}=q$ for some $m$ dividing $n$.

(ii) $\left|C_{\bar{G}_{\sigma}}(\alpha)\right| \geq q^{1 / m}-1$ for some divisor $m$ of $n$. Further, either $q^{1 / m}$ is an integral power of $p$, or $\bar{G}$ has type $B_{2}, F_{4}$ or $G_{2}, p=$ $2,2,3$ respectively, and $q^{2 / m}$ is an odd power of $p$. 
(iii) There is a polynomial $f$ with real coefficients and a positive integer $m$ dividing $n$ such that $\left|f\left(q^{1 / m}\right)\right|$ is an integer dividing $\left|C_{\bar{G}_{\sigma}}(\alpha)\right|$. The degree of $f$ is at most $l$ and its roots are roots of 1 of degree at most $2 l$ over $Q$.

Proof. (i) From Propositions 4.1 and 4.2, we see that $C_{\bar{G}_{\sigma}}(\alpha)$ has the form $C_{\bar{G}}(\beta)_{\psi}$, where $\beta$ is a semisimple algebraic automorphism of $\bar{G}$ and $\psi$ is a Frobenius map on $\bar{G}$ commuting with $\beta$ and so leaving $H=C_{\bar{G}}(\beta)$ invariant. Further, $q(\psi)^{m}=q$, where $m \mid n$. By a well known theorem of Steinberg [18, (8.1), (9.2)], $H$ is an infinite reductive algebraic group.

(ii), (iii) By another theorem of Steinberg [18, (10.1)], $\psi$ leaves invariant some maximal torus $S$ of $H$. The rank of $S$ is at most l. We obtain (ii) and (iii) by applying Lemma 3.3 to $S_{\psi}$. Since $q^{1 / m}=q(\psi)$, it or its square is a power of $p$ as stated.

\section{Proofs of Theorems $A^{\prime}$ and $C$.}

Proof of Theorem $A^{\prime}$. We prove the theorem by induction on $n$. The case $n=1$ is trivial. Note that when Theorem $\mathrm{A}^{\prime}$ has been proved for all divisors of a given integer $n$, then Theorem $\mathrm{A}$ follows for all divisors of $n$, by the proof of that theorem. When we say that a given quantity is bounded during the course of this proof, we mean that it is bounded in terms of $n$ and $k$.

The classification of finite simple groups tells us that our simple group $G$ is either sporadic, alternating, or of Lie type. As there are only finitely many sporadic groups, they can be ignored. The alternating groups will be left as a simple exercise for the reader, and so we now assume that $G$ is of Lie type and use the notation of $\S 3$.

It is sufficient to bound the rank $l$ of $\bar{G}$ and the field parameter $q$ of $G$. Let $\alpha$ be the given automorphism. Write $n=p_{1}^{r_{1}} \cdots p_{t}^{r_{t}}$, where the $p_{i}$ are distinct primes, and let $\alpha=\alpha_{1} \cdots \alpha_{t}$, where $\alpha_{i}$ has order $p_{i}^{r_{i}}$ and the $\alpha_{i}$ commute. Let $\beta_{i}$ be an element of order $p_{i}$ in $\left\langle\alpha_{i}\right\rangle$.

Suppose first that $t=1, p_{1}=p$, and $n=p^{r}$. Then $\alpha$ leaves invariant some Sylow $p$-subgroup $P$ of $G$. Let $\Phi(P)$ be the Frattini subgroup of $P$ and $\bar{P}$ be the elementary abelian group $P / \Phi(P)$. Then $|\bar{P}|$ is divisible by $q^{l}$ (see $\left.[6,5.3,13.6]\right)$. Also $\left|C_{\bar{P}}(\alpha)\right| \leq k \quad([2$, VIII.10.14]). We have $(\alpha-1)^{p^{r}}=0$ on $\bar{P}$, and it follows by well known arguments using Jordan blocks or the like that $|\bar{P}|$ divides $\left|C_{\bar{p}}(\alpha)\right|^{p^{r}} \leq k^{p^{r}}$. Hence $q^{l}$ is bounded, as required. 
Now we may assume without loss of generality that

$$
p_{1} \neq p \text {. }
$$

We may further assume that

$$
\beta_{i} \in \bar{G}_{\sigma} \text { for } 1 \leq i \leq t .
$$

For if some $\beta_{i}$ is not in $\bar{G}_{\sigma}$, then Lemma 3.1 tells us that $C_{G}\left(\beta_{i}\right)$ involves a non-abelian simple group $H$ such that $|G|$ is bounded in terms of $|H|$. But applying induction to the action of $\alpha$ on $C_{G}\left(\beta_{i}\right)$ gives us a bound on $|H|$ and hence on $|G|$.

Now we show that

\section{$\bar{G}$ has bounded rank.}

This is because, if $\bar{G}$ has rank $l$ and classical type, then by Lemma 3.4, $C_{G}\left(\beta_{1}\right)$ involves a group of Lie type of the form $H=O^{p^{\prime}}\left(\bar{H}_{\tau}\right)$, where $\bar{H}$ is a simple algebraic group of rank at least $(l-n) / r(n) \geq$ $(l-n) / 2 n, \tau$ is a Frobenius map on $\bar{H}$, and $H$ is defined over an extension of the field over which $G$ is defined. By induction, we have a bound on $|H|$, and this gives us one on $l$. The ranks of the exceptional groups are of course bounded in any case.

Since $\left|C_{\bar{G}_{\sigma}}(\alpha) / G\right|$ is bounded in terms of $l$ (in fact it is at most $l+1$, see [6, pp. 98-99] for example), we have

$$
\left|C_{\bar{G}_{\sigma}}(\alpha)\right| \text { is bounded . }
$$

It remains to bound $q$. Now if $C_{\bar{G}}\left(\beta_{i}\right)^{0}$ is non-abelian for some $i$ such that $p_{i} \neq p$, then again we find that $C_{G}\left(\beta_{i}\right)$ involves a group in Chev over some extension field of $F_{q}\left(F_{q^{2}}\right.$ if $G$ is a Suzuki or Ree group). This group will be simple unless $q$ is already very small, and we obtain our bound on $q$ by induction. If $p_{i}=p$ for some $i$, then as $\beta_{i} \in C_{\bar{G}}\left(\beta_{1}\right)^{0}$, we have that $C_{\bar{G}}\left(\beta_{1}\right)^{0}$ is non-abelian (see [14, Theorem $\mathrm{C}$ ], for example, for this well known fact ). We conclude that we may assume that

$$
p_{i} \neq p \quad \text { for } 1 \leq i \leq t .
$$

By Theorem 4.4, $\left|C_{\bar{G}_{\sigma}}(\alpha)\right| \geq q^{1 / n}-1$. This bounds $q$ and concludes the proof.

Proof of Theorem $\mathrm{C}$. This is now rather straightforward. Most cases were covered in [11], but we will give here a proof covering all cases. For our simple group $G$ we have, by [14, Lemma 4.3], a simple 
Chevalley group $\bar{G}$ over $K$, a Frobenius map $\sigma$ on $\bar{G}$, and a sequence of integers $n(1)|n(2)| \ldots$ such that

$$
G=\bigcup_{i=1}^{\infty} G_{i}, \quad \text { where } G_{i}=O^{p^{\prime}}(\bar{G})_{\sigma^{n(l)}} .
$$

Let $\Phi$ and $\Gamma$ be the field and graph automorphisms of $\bar{G}$ as at the beginning of $\S 4$. Let $H_{i}=\bar{G}^{\sigma^{(i)}}$ and $H=\bigcup_{i=1}^{\infty} H_{i}$. Then we can take $\sigma \in \Phi \Gamma$ and $\alpha$ to be induced on $G$ by an element $\beta \theta$, where $\beta$ is conjugation by some element $h$ of $H$ and $\theta \in \Phi \Gamma[19$, p. 195]. Further, $\theta$ commutes with $\sigma$. In fact, one of $\theta$ and $\sigma$ will be in $\Phi$, which commutes elementwise with $\Phi \Gamma$. Let $h \in H_{i}$. Then $\beta \theta$ leaves $H_{j}$ invariant if $j \geq i$, and so we may assume that $\beta \theta$ leaves all $H_{i}$ invariant. Further, $(\beta \theta)^{n}$ is trivial on each $H_{i}$. This is because $C_{H_{i}}\left(G_{i}\right)=1$ and so every non-trivial automorphism of $H_{i}$ restricts non-trivially to $G_{i}$.

Thus, we can think of $\alpha$ as an automorphism of order $n$ of $H$ leaving all $H_{i}$ invariant. Since the groups $H_{i} / G_{i}$ have order bounded independently of $i$ (in fact by $l+1$, where $l$ is the rank of $\bar{G}$ ), it will suffice to show that the numbers $\left|C_{H_{t}}(\alpha)\right|$ have infinitely many prime divisors between them.

Let $q=q(\sigma)$ and $l$ be the rank of $\bar{G}$. By Theorem 4.4, $\left|C_{H_{t}}(\alpha)\right|$ is divisible by $\left|f_{i}\left(q^{n(i) / m(i)}\right)\right|$, where $f_{i}$ is some real monic polynomial whose degree is at most $l$ and whose roots are roots of 1 of degree at most $2 l$ over the rationals. The roots of $f_{i}$ are drawn from a finite set, so only finitely many polynomials occur as $f_{i}$. There are clearly only finitely many possibilities for the $m(i)$. Thus, there is an infinite sequence $i_{1}, i_{2}, \ldots$ a polynomial $f$ and a divisor $m$ of $n$ such that $f_{i_{r}}=f$ and $m_{i_{r}}=m$ for all $r$. By [11, Lemma 2.3], with $s=q^{1 / m}$, the numbers $f\left(q^{n(i) / m}\right)$ have infinitely many prime divisors between them. This concludes the proof.

\section{REFERENCES}

[1] M. Aschbacher and G. Seitz, Involutions in Chevalley groups over fields of even order, Nagoya J. Math., 63 (1976), 1-91.

[2] N. Blackburn and B. Huppert, Finite Groups I, II, Springer-Verlag, Berlin, 1982.

[3] A. Borel, Linear Algebraic Groups, Benjamin, New York, 1969.

[4] R. Brauer and P. Fong, On the centralizers of p-elements in finite groups, Bull. London Math. Soc., 6 (1974), 319-324.

[5] R. Brauer and K. A. Fowler, On groups of even order, Ann. of Math., (2) 62 (1955), 565-583. 
[6] R. W. Carter, Simple Groups of Lie Type, Wiley-Interscience, London, 1972.

[7] _ Finite Groups of Lie Type: Conjugacy Classes and Complex Characters, Wiley-Interscience, Chichester, 1985.

[8] J. Dieudonné, La géometrie des Groupes Classiques, Springer-Verlag, Berlin, 1955.

[9] D. Gorenstein and R. Lyons, The local structure of finite groups of characteristic 2 type, Memoirs Amer. Math. Soc., 42 No. 276 (1983).

[10] Morton E. Harris, Finite groups containing an intrinsic 2-component of Chevalley type over a field of odd order, Trans. Amer. Math. Soc., 272 (1982), 1-65.

[11] B. Hartley, Fixed points of automorphisms of certain locally finite groups and Chevalley groups, J. London Math. Soc., (2) 37 (1988), 421-436.

[12] Brian Hartley and Volker Turau, Finite groups admitting an automorphism of prime power order with few fixed points, Math. Proc. Cambridge Philos. Soc., 102 (1987), 431-441.

[13] B. Hartley and I. M. Isaacs, On characters and fixed points of coprime operator groups, J. Algebra, 131 (1990), 342-358.

[14] B. Hartley and M. Kuzucuoğlu, Centralizers of elements in locally finite simple groups, Proc. London Math. Soc., (3) 62 (1991), 301-324.

[15] O. H. Kegel and B. A. F. Wehrfritz, Locally Finite Groups, North-Holland, Amsterdam, 1973.

[16] G. Seitz, The root subgroups for maximal tori in finite groups of Lie type, Pacific J. Math., 106 (1983), 153-244.

[17] T. A. Springer and R. Steinberg, Conjugacy classes, in Seminar on Finite Groups and Related Algebraic Groups, Lecture Notes in Math. Vol. 131, SpringerVerlag, Berlin, 1970, reprinted 1986.

[18] R. Steinberg, Endomorphisms of algebraic groups, Memoirs Amer. Math. Soc., 80 (1968).

[19] $\frac{}{1967}$, Lectures on Chevalley groups, Mimeographed notes, Yale University, 1967.

Received August 30, 1990 and in revised form March 6, 1991.

UNIVERSITY OF MANCHESTER

OXFORD ROAD

MANCHester M13 9PL, ENGLAND 



\title{
PACIFIC JOURNAL OF MATHEMATICS EDITORS
}

\author{
V. S. VARADARAJAN \\ (Managing Editor) \\ University of California \\ Los Angeles, CA 90024-1555-05 \\ Herbert Clemens \\ University of Utah \\ Salt Lake City, UT 84112 \\ F. Michael Christ \\ University of California \\ Los Angeles, CA 90024-1555 \\ THOMAS ENRIGHT \\ University of California, San Diego \\ La Jolla, CA 92093
}

Nicholas ERcolani

University of Arizona

Tucson, AZ 85721

R. FINN

Stanford University

Stanford, CA 94305

VAUGHAN F. R. JoNeS

University of California

Berkeley, CA 94720

C. C. MOORE

University of California

Berkeley, CA 94720

MARTIN SchaRLEMANN

University of California

Santa Barbara, CA 93106

HAROLd StaRK

University of California, San Diego

La Jolla, CA 92093

SteVen Kerckhoff

Stanford University

Stanford, CA 94305

\section{ASSOCIATE EDITORS}

\begin{tabular}{|c|c|c|c|c|}
\hline R. ARENS & $\begin{array}{l}\text { E. F. BECKENBACH } \\
(1906-1982)\end{array}$ & NeumanN & $\begin{array}{c}\text { F. WoLF } \\
(1904-1989)\end{array}$ & K. YoshidA \\
\hline \multicolumn{5}{|c|}{ SUPPORTING INSTITUTIONS } \\
\hline \multicolumn{2}{|c|}{ JNIVERSITY OF ARIZONA } & \multicolumn{3}{|c|}{ UNIVERSITY OF OREGON } \\
\hline \multicolumn{2}{|c|}{ UNIVERSITY OF BRITISH COLUMBIA } & \multicolumn{3}{|c|}{ UNIVERSITY OF SOUTHERN C } \\
\hline \multicolumn{2}{|c|}{ CALIFORNIA INSTITUTE OF TECHNOLOGY } & \multirow{2}{*}{\multicolumn{3}{|c|}{ STANFORD UNIVERSITY }} \\
\hline \multicolumn{2}{|c|}{ UNIVERSITY OF CALIFORNIA } & & & UNIVERSITY OF HAWAII \\
\hline \multicolumn{2}{|c|}{ MONTANA STATE UNIVERSITY } & \multicolumn{3}{|c|}{ UNIVERSITY OF TOKYO } \\
\hline \multicolumn{2}{|c|}{ UNIVERSITY OF NEVADA, RENO } & \multicolumn{3}{|c|}{ UNIVERSITY OF UTAH } \\
\hline \multicolumn{2}{|c|}{ NEW MEXICO STATE UNIVERSITY } & \multicolumn{3}{|c|}{ DN STATE UNIVERSITY } \\
\hline \multicolumn{2}{|c|}{ OREGON STATE UNIVERSITY } & UNIVER & OF WASHIN & \\
\hline
\end{tabular}




\section{Pacific Journal of Mathematics}

Vol. 152, No. $1 \quad$ January, 1992

B. V. Rajarama Bhat, On a characterization of velocity maps in the space of observables .......................................

John David Brillhart, Note on the discriminant of certain cyclotomic period

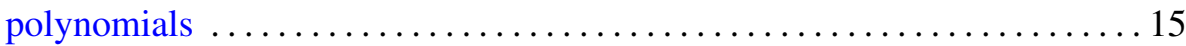

Steven R. Costenoble and Stefan Waner, The equivariant Thom

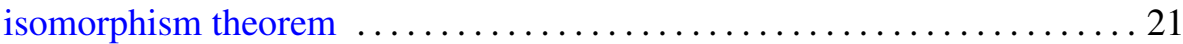

Karl Heinz Dovermann and Dong Youp Suh, Smith equivalence for finite

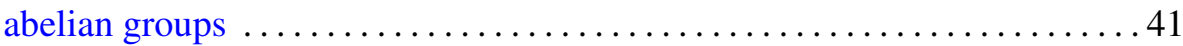

Klaus-Jochen Engel, On singular perturbations of second order Cauchy

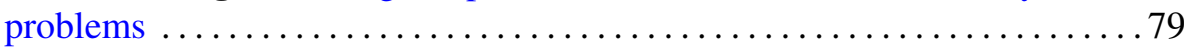

Angel Ferrandez and Pascual Lucas, On surfaces in the 3-dimensional

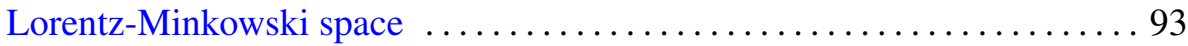

Brian Hartley, A general Brauer-Fowler theorem and centralizers in locally

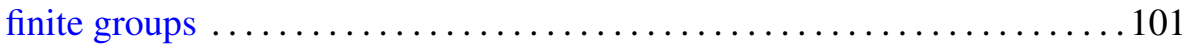

Joel Hass, Intersections of least area surfaces .................... 119

Edward Kissin, Indices of unbounded derivations of $C^{*}$-algebras $\ldots \ldots \ldots 125$

Erhard Luft and Denis Karmen Sjerve, On regular coverings of

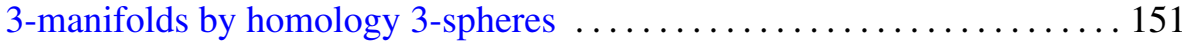

Daniel Pecker, Sur la projection de variétés algébriques réelles .......... 165

Claus Scheiderer, Some remarks on orderings under finite field

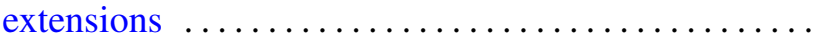

Fernando Serrano, Elliptic surfaces with an ample divisor of genus two 